\title{
Potential antitumor agent from the endophytic fungus Pestalotiopsis photiniae induces apoptosis via the mitochondrial pathway in HeLa cells
}

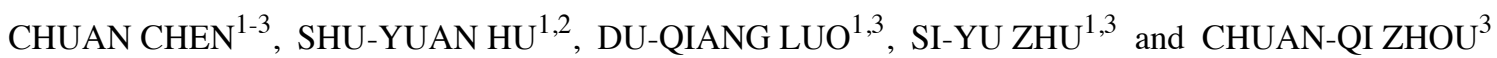 \\ ${ }^{1}$ College of Life Science, Hebei University, Baoding, Hebei 071002; ${ }^{2}$ Key Laboratory of Microbial Diversity Research and \\ Application of Hebei Province, Baoding, Hebei 071002; ${ }^{3}$ College of Life Science, Key Laboratory of Medicinal Chemistry \\ and Molecular Diagnosis of the Ministry of Education, Hebei University, Baoding, Hebei 071002, P.R. China
}

Received February 1, 2013; Accepted April 30, 2013

DOI: $10.3892 /$ or.2013.2618

\begin{abstract}
Dimethylallyloxy)-5-methyl-6-methoxyphthalide (DMMP) has previously been isolated from the endophytic fungus Pestalotiopsis photiniae. Although the cytotoxic activities of DMMP have been reported, little is known concerning the molecular mechanism of its cytotoxic effect. In the present study, we investigated the effect of DMMP on the growth of several types of cancer cell lines and investigated the mechanism of its antiproliferative effect. DMMP caused the growth inhibition of human cancer lines HeLa, MCF7 and MDA-MB-231, but had little antiproliferative effect on MRC5 normal lung cells. DMMP also significantly caused cell cycle arrest in the G1 phase and upregulated the cyclin-dependent kinase inhibitor p $27^{\mathrm{KIPI}}$ protein in the $\mathrm{HeLa}$ cells. Moreover DMMP was able to induce marked nuclear apoptotic morphology in HeLa cells. DMMP induced apoptosis and loss of mitochondrial membrane potential $(\Delta \Psi \mathrm{m})$ in the HeLa cells. Although the activated forms of caspase-9 and -3 in HeLa cells were detected, pretreatment with caspase inhibitors (Ac-DEVD-CHO and Z-VAD-FMK) failed to attenuate DMMP-induced cell death. In addition, protein levels of the p53 family members, p53 and p73, were upregulated, and DMMP significantly increased the mRNA expression of proapoptotic Bcl-2 family genes (PUMA, NOXA, Bax, Bad and Bim). HPV E6-E7 mRNA levels were reduced. In conclusion, DMMP demonstrates potential for use in the treatment of cervical cancer.
\end{abstract}

Correspondence to: Professor Du-Qiang Luo, College of Life Science, Key Laboratory of Medicinal Chemistry and Molecular Diagnosis of the Ministry of Education, Hebei University, Wusi East Street, Baoding, Hebei 071002, P.R. China

E-mail: duqiangluo@163.com

Key words: 4-(3',3'-dimethylallyloxy)-5-methyl-6-methoxyphthalide, apoptosis, HeLa cells, mitochondrial pathway

\section{Introduction}

Natural products and their derivatives have historically been invaluable as sources of chemopreventive and therapeutic agents (1). Recently, more attention has been paid to bioactive natural products of endophytic fungi, isolated from higher plants (2). Endophytic fungi are important components of microbial biodiversity. Biological diversity means chemical diversity implying unique structural features and pronounced biological activities as lead structures for novel drugs (3).

Apoptotic cell death is a self-destructing process under strict control and plays an important role in tissue homeostasis. Impairment of the apoptotic signaling pathway leads to insufficient or excessive cell death which results in the progression of human cancers (4). Thus, a chemical compound which promotes cell apoptosis and inhibits cancer cell proliferation is considered to be a potential anticancer drug (5).

The p53 family members are mainly involved in apoptosis induction and cell cycle arrest under cellular stress. The p53 family consists of 3 members: p53, p63 and p73. Tumor suppressor $\mathrm{p} 53$ is a transcription factor that maintains genome stability and normal cell growth $(6,7)$. The tumor suppressor p53 is activated in response to stress such as DNA damage and oncogene activation. p53 can promote cell apoptosis and thus enhance the chemosensitivity to anticancer agents $(8,9)$. Unlike p53, p73 is rarely mutated in human cancers. Due to high homology to p53, the p53-related protein p73 is capable of transactivating p53 target genes and also plays an important role in the regulation of chemosensitivity $(10,11)$. The p53 family members are an attractive pharmaceutical target for cancer therapy. Many compounds have been discovered which can induce the activation of p53 and the death of tumor cells $(12-15)$.

4-(3',3'-Dimethylallyloxy)-5-methyl-6-methoxyphthalide (DMMP) was initially found in the liquid culture of the fungus Alternaria porri (Fig. 1) (16,17). DMMP was reported to have antifungal activity and cytotoxic activity in cancer cell lines. In our research, DMMP was isolated from the plant endophytic fungus Pestalotiopsis photiniae (18). Although the cytotoxic activities of DMMP have been reported, little is known concerning the molecular mechanism of its cytotoxic effect. 


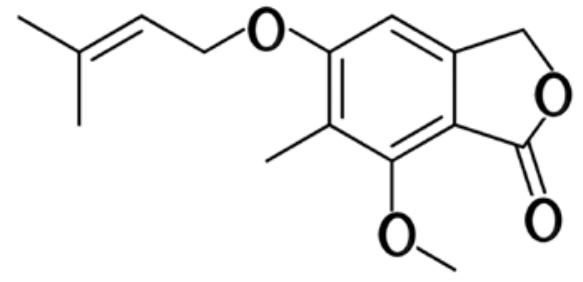

Figure 1. Structure of 4-(3',3'-dimethylallyloxy)-5-methyl-6-methoxyphthalide (DMMP).

Here, for the first time, we discovered that DMMP inhibits the growth of several cancer cell lines and we investigated the mechanism of its antiproliferative effect. These results were significant in that they provide a mechanistic framework for further exploration of the use of DMMP as a novel antitumor agent.

\section{Materials and methods}

Materials. Caspase inhibitor Z-VAD-FMK, caspase-3 specific inhibitor Z-DEVD-FMK, PD9805 were from Sigma-Aldrich (St. Louis, MO, USA; MTT, acridine orange and ethidium bromide were from Amresco LLC (Solon, OH, USA). JC-1 was from Beyotime Institute of Biotechnology (Jiangsu, China). Rabbit polyclonal antibodies against Fas-L, Fas, p73, p53, Bax, Bcl-2, survivin, CDK2, Akt, p-AKT (Thr308), ERK, p-ERK1/2 (Thr202/Tyr204), p-RB (Ser807/811), GAPDH, Bid, caspase-8, caspase-9, cyclin E, p27 ${ }^{\mathrm{KIPI}}$, Bcl-xL and E2F1 were obtained from Santa Cruz Biotechnology, Inc. (Santa Cruz, CA, USA). Mouse monoclonal p53 and mouse monoclonal CDK6 were obtained from Santa Cruz Biotechnology, Inc., and rabbit polyclonal activated caspase-3 was from Cell Signaling Technology, Inc. (Danvers, MA, USA). MEM/NEAA medium, DMEM, L15 medium were from Invitrogen Life Technologies (Carlsbad, CA, USA). Propidium iodide (PI)/RNase staining buffer and the Annexin V-FITC Apoptosis Detection kit were from BD Pharmingen (San Diego, CA, USA). PVDF membranes were from Millipore (Billerica, MA, USA) and the PrimeScript ${ }^{\mathrm{TM}}$ High Fidelity RT-PCR kit and SYBR ${ }^{\circledR}$ Premix Dimer Eraser were from Takara Bio, Inc. (Shiga, Japan). DMMP (Fig. 1) was provided by our research group at Hebei University (purity $>99 \%$, HPLC analysis) (18).

Cell culture. All cell lines were purchased from Cell Resource Center, IBMS, CAMS/PUMC. MCF7 human breast adenocarcinoma and HeLa human cervical cancer cells were grown in Dulbecco's modified Eagle's medium (DMEM) supplemented with $10 \%$ heat-inactivated fetal bovine serum (FBS) (Invitrogen Life Technologies). MRC5 normal lung cells were grown in MEM/NEAA supplemented with $10 \%$ heat-inactivated FBS. MDA-MB-231 human breast adenocarcinoma cells were grown in L15 medium supplemented with $10 \%$ heat-inactivated FBS and $1 \%$ glutamine. All cell lines were cultured at $37^{\circ} \mathrm{C}$ in a humidified incubator containing $5 \% \mathrm{CO}_{2}$.

MTT assay. Cell viability was determined using the colorimetric 3-(4,5-dimethylthiazol-2-yl)-2,5-diphenyl tetrazolium bromide (MTT) assay. Exponentially growing cells
$(8,000$ cells/well) were seeded in 96 -well plates and cultured at $37^{\circ} \mathrm{C}$, under $5 \% \mathrm{CO}_{2}$ and $95 \%$ air for $24 \mathrm{~h}$. Cells were treated with DMMP at different concentrations $(5,10,20,40$ and $80 \mu \mathrm{g} / \mathrm{ml}$ dissolved in DMSO) for different time periods. The DMSO concentration was maintained below $0.5 \%$ which was found to have no antiproliferative effect on the cell lines. Then $20 \mu 1$ MTT $(5 \mathrm{mg} / \mathrm{ml})$ was added to each well. After incubation at $37^{\circ} \mathrm{C}$ for $4 \mathrm{~h}, 100 \mu 110 \%$ SDS- $\mathrm{HCl}$ was added and incubated at $37^{\circ} \mathrm{C}$ overnight. The $\mathrm{OD}$ value of the system was measured at a wavelength of $570 \mathrm{~nm}$. Five wells were counted in 3 different experiments. The 50\% inhibitory concentration $\left(\mathrm{IC}_{50}\right)$ of DMMP on cells was calculated by the MTT assay.

Inhibitor treatment. The cell culture was preincubated for $2 \mathrm{~h}$ with one of the following inhibitors: the cell-permeable pancaspase inhibitor Z-VAD-FMK $(10 \mu \mathrm{M})$, the caspase-3-specific inhibitor Z-DEVD-FMK $(10 \mu \mathrm{M})$ or ERK inhibitor PD98059 $(20 \mu \mathrm{M})$ before the addition of DMMP. Cell viability was measured by the MTT assay.

Caspase activity assay. Caspase activation was measured using a caspase colorimetric assay kit (Beyotime Institute of Biotechnology). Briefly, following cell treatment with $40 \mu \mathrm{g} / \mathrm{ml}$ DMMP for the indicated periods of time, cells were harvested and $25 \mu \mathrm{l}$ of cold lysis buffer $/ 10^{6}$ cells was added. The cell lysates were incubated on ice for $10 \mathrm{~min}$ and then centrifuged at $16,000 \mathrm{x}$ g for $15 \mathrm{~min}$. The protein concentration was determined by Bradford assay. To each reaction well, $10 \mu \mathrm{l}$ of caspase-3 fluorogenic substrate (Ac-DEVD-pNA) was added to $10 \mu \mathrm{l}$ sample (1-3 mg/ml) and $80 \mu \mathrm{l}$ detection buffer. The plate was incubated at $37^{\circ} \mathrm{C}$ for $2 \mathrm{~h}$. The plate was read on a fluorescence microplate reader.

Acridine orangelethidium bromide (AO/EB) staining. Exponentially growing cells were seeded on polylysine-coated glass coverslip in a 24 -well-plate and cultured at $37^{\circ} \mathrm{C}$, under $5 \% \mathrm{CO}_{2}$ for $24 \mathrm{~h}$. After incubation with $40 \mu \mathrm{g} / \mathrm{ml}$ DMMP, cells were washed with PBS for 3 times and then stained with $100 \mu \mathrm{g} / \mathrm{ml} \mathrm{AO} / \mathrm{EB}$ for $5 \mathrm{~min}$. Coverslips were mounted and the fluorescence was visualized using fluorescence microscopy (Olympus).

JC-1 analysis. Cells $\left(10^{4}\right)$ were seeded on polylysine-coated glass coverslips in a 24 -well plate, and incubate at $37^{\circ} \mathrm{C}$ under $5 \% \mathrm{CO}_{2}$ for $24 \mathrm{~h}$. After being exposed to DMMP for 12 and $24 \mathrm{~h}$, cells were washed with PBS for 3 times, and then incubated with a final concentration of $2 \mu \mathrm{M} \mathrm{JC}-1$ dye at $37^{\circ} \mathrm{C}$ under $5 \% \mathrm{CO}_{2}$ for $20 \mathrm{~min}$. Cells were treated with a final concentration of $50 \mu \mathrm{M} \mathrm{CCCP}$ at $37^{\circ} \mathrm{C}$ for $20 \mathrm{~min}$ as a positive control. After 3 washes with PBS, the coverslips were mounted and the fluorescence was visualized using fluorescence microscopy (Olympus).

Flow cytometric assay. HeLa cells were treated with DMMP at a concentration of $40 \mu \mathrm{g} / \mathrm{ml}$ for 12,24 and $36 \mathrm{~h}$. Cells $\left(10^{6}\right)$ were collected by centrifuging at $1,000 \mathrm{rpm}$ for $5 \mathrm{~min}$, and then cells were washed twice with ice-cold PBS. For cell cycle analysis, cells were fixed in ice-cold ethanol $(70 \% \mathrm{v} / \mathrm{v})$ and stained with $0.5 \mathrm{ml} \mathrm{PI/RNase} \mathrm{staining} \mathrm{buffer} \mathrm{(BD}$ Pharmingen) for $15 \mathrm{~min}$ at room temperature and analyzed 
by flow cytometry (Becton-Dickinson, Franklin Lakes, NJ, USA). Apoptotic/necrotic cells were detected using the Annexin V-FITC Apoptosis Detection kit (BD Pharmingen). Briefly, cells were incubated with binding buffer $(10 \mathrm{mM}$ HEPES/NaOH, pH 7.5, $140 \mathrm{mM} \mathrm{NaCl}$ and $2.5 \mathrm{mM} \mathrm{CaCl}_{2}$ ) and stained with PI and FITC-labeled Annexin V for 15 min at room temperature in the dark. Cell fluorescence was evaluated by flow cytometry using a FACSCalibur instrument and analyzed by Cell Quest software.

Western blotting. Western blotting was used to determine the expression of various key proteins. Cells were seeded in a culture bottle at $37^{\circ} \mathrm{C}$ under $5 \% \mathrm{CO}_{2}$ and $95 \%$ air. The cells were treated with DMMP $(40 \mu \mathrm{g} / \mathrm{ml}$ for 12,24 and $36 \mathrm{~h})$. Approximately $2 \times 10^{6}$ cells were collected and washed with ice-cold PBS. Subsequently, cells were incubated in lysis buffer (50 mM HEPES-NaOH, $100 \mathrm{mM} \mathrm{NaCl}, 0.5 \% \mathrm{NP}-40$, $2.5 \mathrm{mM}$ EDTA, $10 \%$ glycerol, $1 \mathrm{mM}$ DTT, $1 \mathrm{mM} \mathrm{Na}_{3} \mathrm{VO}_{4}$, $5 \mathrm{mM}$ NaF, $1 \mathrm{mM}$ PMSF, $2 \mu \mathrm{g} / \mathrm{ml}$ pepstatin, $5 \mu \mathrm{g} / \mathrm{ml}$ leupetin, $2 \mu \mathrm{g} / \mathrm{ml}$ aprotintin) for $10 \mathrm{~min}$ on ice, collected by scraping and kept on ice for $30 \mathrm{~min}$. Finally, after freezing and thawing, cells were centrifuged at $14,000 \mathrm{rpm}$ for $30 \mathrm{~min}$ at $4^{\circ} \mathrm{C}$. Cell lysates were centrifuged at $12,000 \mathrm{rpm}$ for $15 \mathrm{~min}$ at $4^{\circ} \mathrm{C}$. Protein concentrations in lysates were determined by Bradford assay. Fifty micrograms of protein from the protein lysates per sample was denatured in $2 \mathrm{X}$ sample buffer and was loaded on sodium dodecyl sulfate-polyacrylamide (SDS) gel electrophoresis on 8-12\% Tris-glycine gel. After electrophoresis, proteins were transferred onto PVDF membranes (Millipore) followed by blocking with $5 \%$ non-fat milk powder $(\mathrm{w} / \mathrm{v})$ in Tris-buffered saline [10 mM Tris- $\mathrm{HCl}$ (pH 7.5), $100 \mathrm{mM} \mathrm{NaCl}$, $0.1 \%$ Tween-20] for $1 \mathrm{~h}$ at room temperature. The membranes were then incubated with specific primary antibodies for $1 \mathrm{~h}$. After washing, the membranes were incubated with appropriate secondary HRP-conjugated antibodies and visualized by ECL. Each membrane was stripped and reprobed with anti-GADPH antibody to ensure equal protein loading.

Real-time RT-PCR. Approximately $10^{6}$ cells were harvested at the indicated time points and total RNA was isolated using TRIzol reagent (Invitrogen Life Technologies) as described by the manufacturer. The integration of RNA was detected by agarose gel analysis and spectrophotometry. Reverse transcription of total RNA was performed by PrimeScript ${ }^{\mathrm{TM}}$ High Fidelity RT-PCR kit (Takara Bio, Inc). Real-time RT-PCR was performed using a Bio-Rad (Hercules, CA, USA) iCycler PCR machine. Each PCR mixture contained 100 ng of cDNA template and primers at a concentration of $10 \mu \mathrm{M}$ in a final volume of $25 \mu 1$ of SYBR Premix Dimer Eraser. Specificity of each PCR was examined by the melting temperature profiles of the final products. Standard curves were calculated using cDNA to determine the linear range and efficiency of each primer pair (primer sequences are provided upon request). Reactions were conducted in triplicate, and relative amounts of gene were normalized to GAPDH. The relative gene expression data were analyzed by the comparative CT method $(\Delta \Delta \mathrm{CT}$ method).

Statistical analysis. The statistical significance of the differences between the control and treated groups was determined

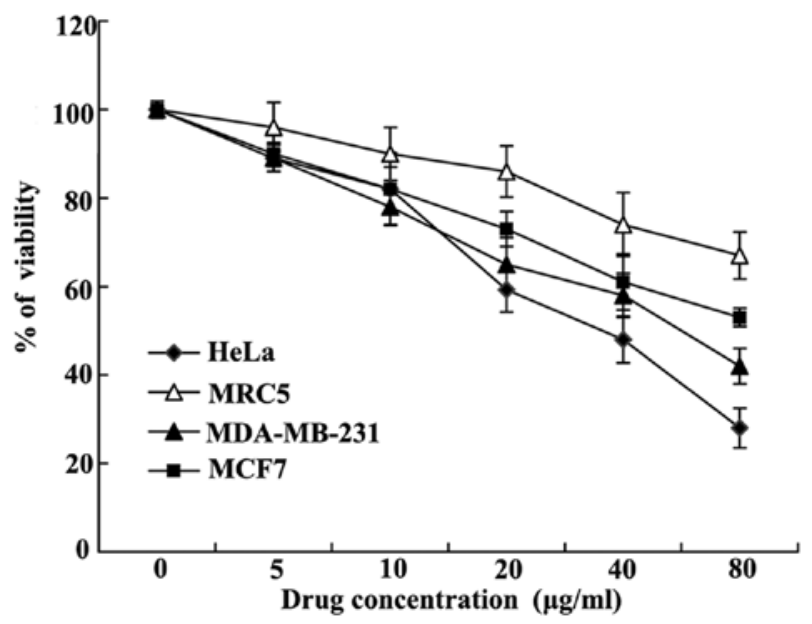

Figure 2. Effect of DMMP on the viability of different cell lines. Cell proliferation was performed using the MTT assay. Cells were treated with 5, 10, 20,40 and $80 \mu \mathrm{g} / \mathrm{ml}$ DMMP or with DMSO $(0.5 \%)$ as the vehicle control for $24 \mathrm{~h}$. The data values are expressed as the means \pm SD of 3 independent experiments.

using the 2-tailed Student's t-test. P-values $<0.05$ were considered to indicate a statistically significant result.

\section{Results}

Effect of DMMP on cell proliferation. To assess the effect of DMMP on cell proliferation, human cancer cell lines, HeLa, MCF7 and MDA-MB-231, and the normal lung fibroblast cell line MRC5 were treated with increasing concentrations of DMMP at $24 \mathrm{~h}$, and cell viability was assessed using the MTT assay (Fig. 2). $\mathrm{IC}_{50}$ for HeLa, MDA-MB-231, MCF7 and MRC5 cells were 36, 51, 81 and $147 \mu \mathrm{g} / \mathrm{ml}$, respectively. DMMP inhibited the proliferation of the cancer cells in a concentration-dependent manner. DMMP showed the most antiproliferative effect on the HeLa cell line. While the normal lung fibroblast cell line MRC5 exhibited less sensitivity. DMMP exhibited slight selective cytotoxic effect.

Effect of DMMP on cell cycle progression. The effect of DMMP treatment on cell cycle arrest was examined.Compared to DMSO treatment, cells treated with DMMP at the indicated times notably accumulated in the G1 phase of the cell cycle with a reduction in the percentage of cells in $\mathrm{S}$ phase (Fig. 3A). DMMP treatment for $24 \mathrm{~h}$ increased the percentage of cells in the G1 phase from 68.38 to $92.0 \%$. These results suggest that DMMP inhibited cellular proliferation of HeLa cells via the G1 phase arrest of the cell cycle.

The expression of cell cycle-regulating proteins was examined. Expression levels of cyclin E were gradually increased after DMMP treatment, with slight upregulation of CDK2 (Fig. 3B). p2 $7^{\mathrm{KIP} 1}$ was able to directly bind several different classes of cyclins and CDKs leading to cell cycle arrest in the G1 phase (19). As shown in Fig. 3B, p2 $7^{\mathrm{KIPl}}$ was dramatically upregulated at $12 \mathrm{~h}$ and this effect increased over time. $\mathrm{pRb}$ is an important regulator of $\mathrm{G} 1 / \mathrm{S}$ phase transition. Phosphorylated pRb was slightly increased (Fig. 3B). E2F1, the binding partner of $\mathrm{pRb}$, was increased at 12 and $24 \mathrm{~h}$. 
A

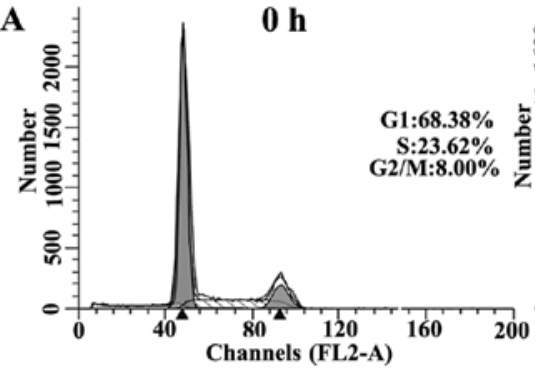

A
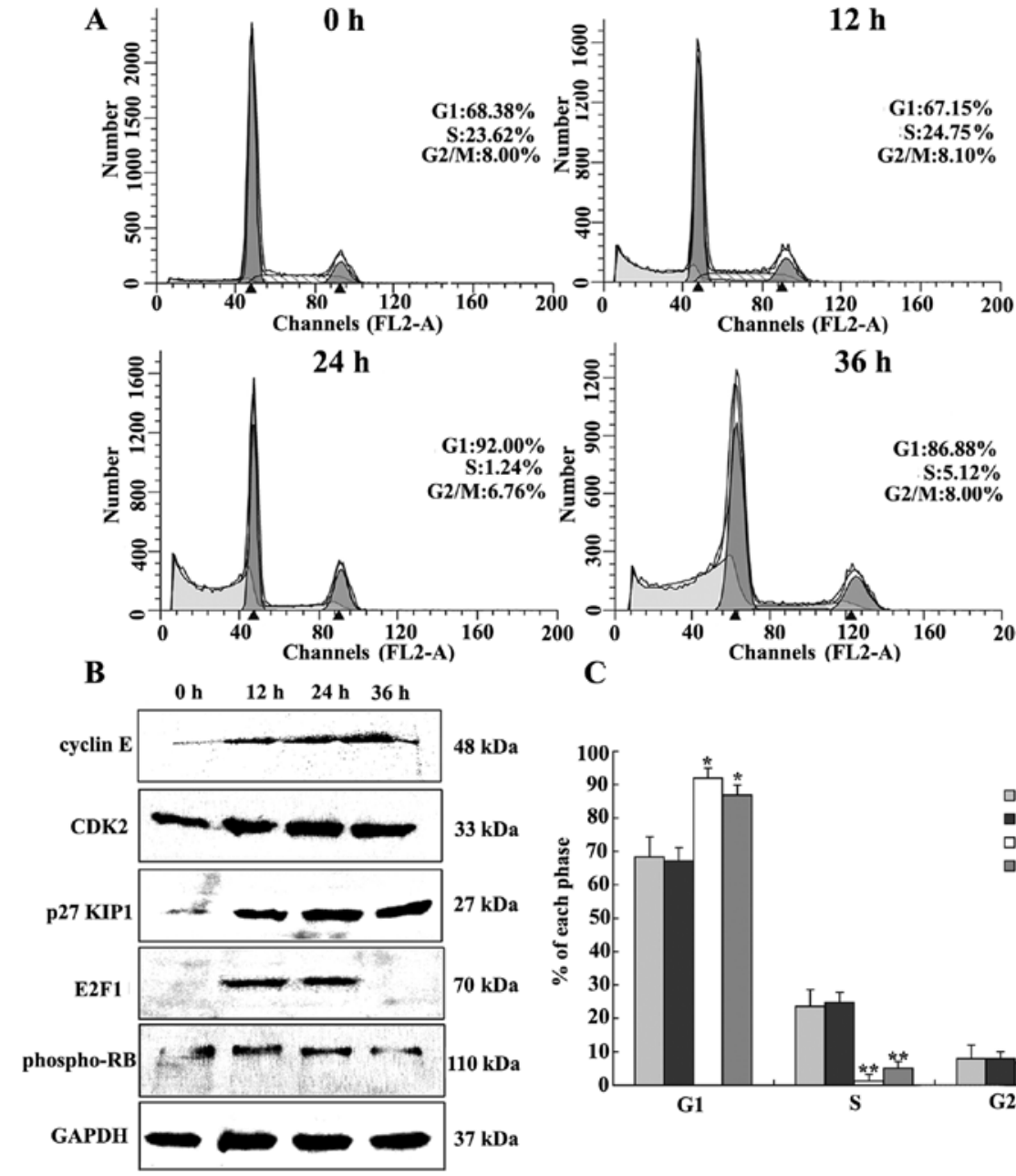

$36 \mathrm{~h}$

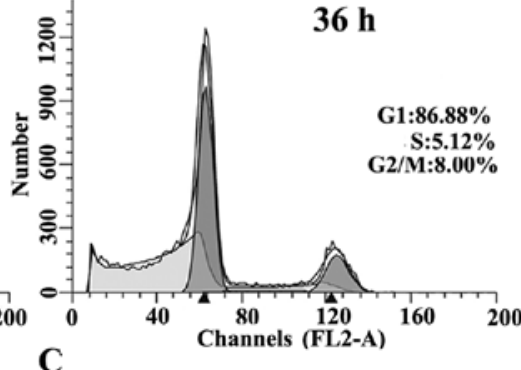

C

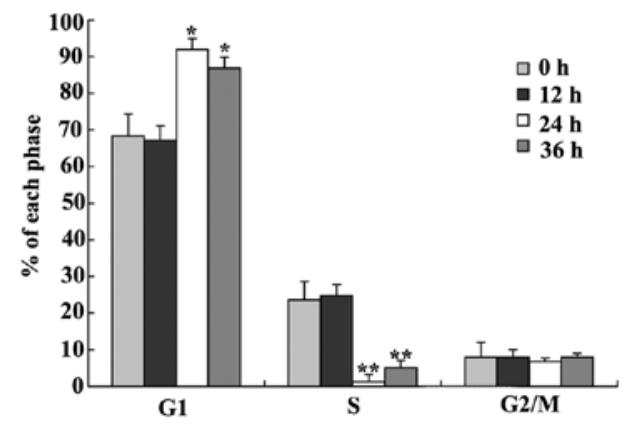

Figure 3. DMMP causes cell cycle arrest in the G1 phase. (A) HeLa cells were treated with DMSO (0.5\%) or DMMP (40 $\mu$ g/ml) for the indicated times and stained with propidium iodide (PI). The DNA content was analyzed using flow cytometry with Cell Quest software. (B) Effect of DMMP on cell cycle regulatory proteins. HeLa cells were treated with $40 \mu \mathrm{g} / \mathrm{ml}$ DMMP for different times. Cell lysates were prepared and western blot analysis was carried out for the expression levels of cell-cycle regulatory proteins, cyclin E, CDK2 and p27 ${ }^{\mathrm{KIP} 1}$. GAPDH was used as a loading control. (C) The histogram of the flow cytometric analysis of HeLa cells. Results are representative of 3 independent experiments. ${ }^{*} \mathrm{P}<0.05,{ }^{* *} \mathrm{P}<0.01$.

DMMP induces apoptosis in HeLa cancer cells. To further investigate the mechanism of the inhibitory effect of DMMP, acridine orange/ethidium bromide $(\mathrm{AO} / \mathrm{EB})$ staining was used to visualize nuclear changes and apoptotic body formation which are characteristic of apoptosis. AO is a membranepermeable dye that can pass through the membrane of intact cells and embed in the nuclear DNA emitting bright green fluorescence. EB can pass through the membrane of a dead or dying cell with orange fluorescence. After HeLa cells were exposed to DMMP for $12 \mathrm{~h}$, early apoptotic cells with green pyknotic-like nuclear chromatin were noted. At $24 \mathrm{~h}$, late apoptotic cells with pyknotic-like deep orange nuclear chromatin were detected. Control cells with normal morphology showed green fluorescence (Fig. 4A). The results suggest that DMMP was able to induce marked apoptotic morphology in HeLa cells in a time-dependent manner.

To investigate the kinetics of the apoptotic cells induced by DMMP, HeLa cells were treated with DMMP for various periods of time, and the apoptotic cell death was analyzed by flow cytometry using Annexin V-PI staining. The proportion of early apoptotic cells (Annexin V-positive and PI-negative) was significantly increased from $2.63 \%$ in the control cells to
$8.52 \%$ at $12 \mathrm{~h}, 38.09 \%$ at $24 \mathrm{~h}$, and $44.46 \%$ at $36 \mathrm{~h}$ following DMMP treatment (Fig. 4B and C). These results indicate that cell death induced by DMMP was mainly caused by induction of apoptosis in a time-dependent-manner.

Caspase activity is induced by DMMP. We investigated whether DMMP induces HeLa cell apoptosis through the caspase pathway. Caspase- $8,-9$ and -3 were detected by western blotting as shown in Fig. 5A. Following DMMP treatment full-length caspase-9 was decreased in a time-dependent manner. Cleaved caspase- 9 was detected indicating activation of caspase- 9 and cleaved caspase- 3 was also detected, while activated caspase- 8 was not detected (Fig. 5A). Meanwhile, expression of the death receptor, Fas, was unchanged and its ligand, FasL was slightly increased (Fig. 5A). Survivin expression was gradually increased and peaked at $24 \mathrm{~h}$ and AIF was also slightly increased in DMMP-induced apoptotic cell death (Fig. 5A).

Caspase-3 can be activated either in the intrinsic or the extrinsic apoptotic pathway. To further identify whether caspase-3 is involved in DMMP-induced apoptosis, the catalytic activity of caspase- 3 was measured using a caspase 
$\mathbf{A}$

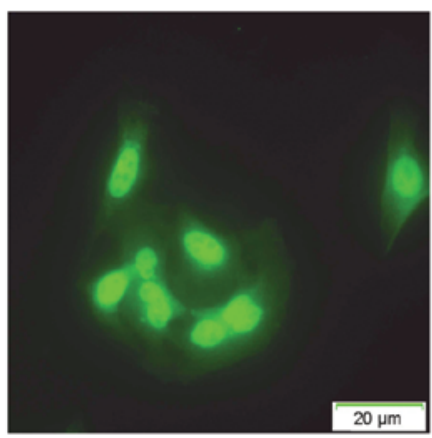

$\mathbf{0 ~ h}$

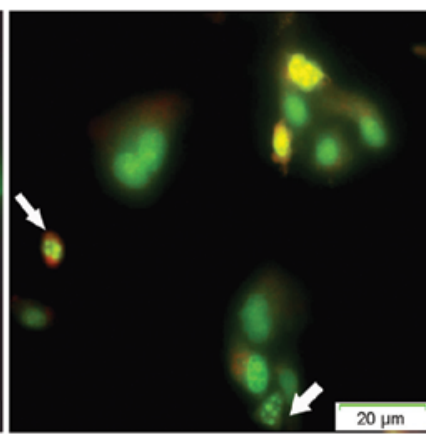

$12 \mathrm{~h}$

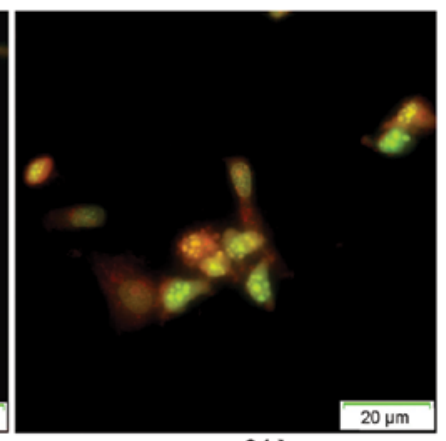

24 h

B
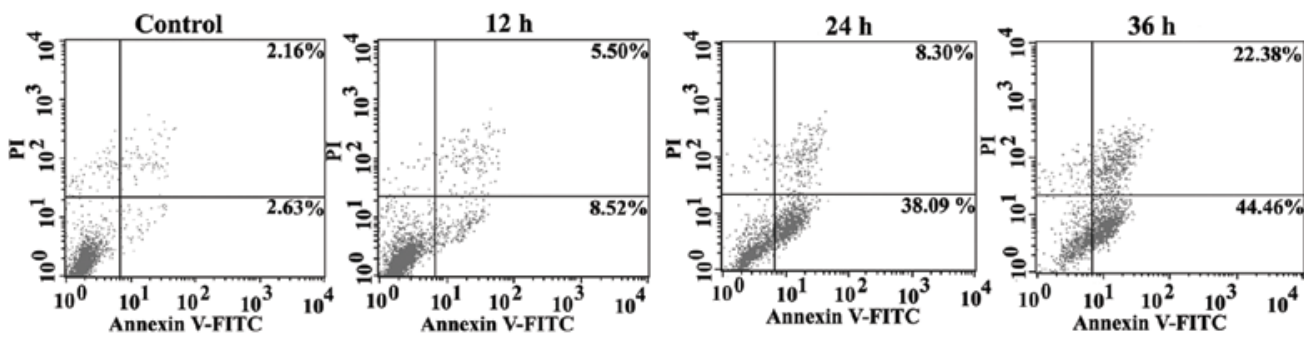

C

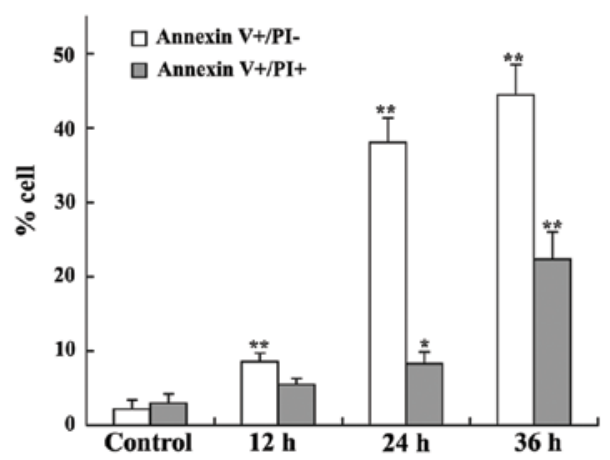

Figure 4. DMMP induces apoptosis in HeLa cancer cells. (A) Acridine orange/ethidium bromide (AO/EB) staining. After incubation with $40 \mu$ g/ml DMMP, cells were washed with PBS for 3 times and then stained with $100 \mu \mathrm{g} / \mathrm{ml} \mathrm{AO} / \mathrm{EB}$ for 5 min. Cell morphology was observed using fluorescence microscopy. The arrows indicate that the nuclei are pyknotic and fragmented. (B) HeLa cells were treated with $40 \mu \mathrm{g} / \mathrm{ml} \mathrm{DMMP}$ for $0,12,24$ and $36 \mathrm{~h}$, then analyzed for apoptosis by flow cytometry. Annexin V and propidium iodide (PI) staining was carried out as described in the Materials and methods section. The control was treated with 0.5\% DMSO. Each value represents the average of 3 independent experiments. (C) Histogram of the flow cytometric analysis of apoptotic cells. Early apoptotic cells (white bars) were Annexin $\mathrm{V}^{+} / \mathrm{PI}^{-}$, whereas late apoptotic/necrotic cells (grey bars) were Annexin $\mathrm{V}^{+} / \mathrm{PI}^{+}$. Values are expressed as means \pm SD of 3 experiments. Significant difference from control value is indicated; ${ }^{*} \mathrm{P}<0.05,{ }^{* *} \mathrm{P}<0.01$.

colorimetric assay kit. Caspase activity was not significantly increased when compared to the control after DMMP treatment (Fig. 5B). To further evaluate the role of caspases in DMMP-induced apoptosis pathway, HeLa cells were pretreated in the presence of the broad-spectrum caspase inhibitor (Z-VAD-FMK) and caspase-3 inhibitor (Ac-DEVD-CHO) for $2 \mathrm{~h}$ before drug treatment. Pretreatment of caspase- 3 and broad-spectrum caspase inhibitors failed to attenuate cell death following DMMP treatment (Fig. 5C). These results showed that DMMP-induced apoptosis may be through a caspase-independent mechanism.

DMMP induces loss of mitochondrial transmembrane potential $(\Delta \Psi m)$. Loss of mitochondrial transmembrane potential is one of several key events that occur in the mitochondria during apoptosis. JC-1 is the most commonly used fluorescent probe to detect the change in mitochondrial membrane potential
$(\Delta \Psi \mathrm{m})$. In healthy cells with high $\Delta \Psi_{\mathrm{m}}, \mathrm{JC}-1$ spontaneously shows intense red fluorescence and in contrast in apoptotic or unhealthy cells with low $\Delta \Psi \mathrm{m}, \mathrm{JC}-1$ shows only green fluorescence. In untreated HeLa cells, JC-1 showed intense red fluorescence. At $12 \mathrm{~h}$ after drug treatment, green fluorescence was noted in some cells indicating the dissipation of $\Delta \Psi \mathrm{m}$. At $24 \mathrm{~h}$, most cells exhibited green fluorescence (Fig. 6). The time course analysis revealed that significant numbers of cells following treatment with DMMP lost $\Delta \Psi \mathrm{m}$.

Effect of DMMP on the Bcl-2 family. Bcl-2 family proteins are key regulators of mitochondrial permeability. Therefore, we investigated whether apoptosis in HeLa cells induced by DMMP is modulated by Bcl-2 family members. Western blotting results revealed that pro-survival Bcl-2 family proteins, $\mathrm{Bcl}-2$, was decreased at $36 \mathrm{~h}$, and $\mathrm{Bcl}-\mathrm{xL}$ was decreased at 12 and $24 \mathrm{~h}$ (Fig. 5A). Bcl-2 mRNA expression significantly increased 

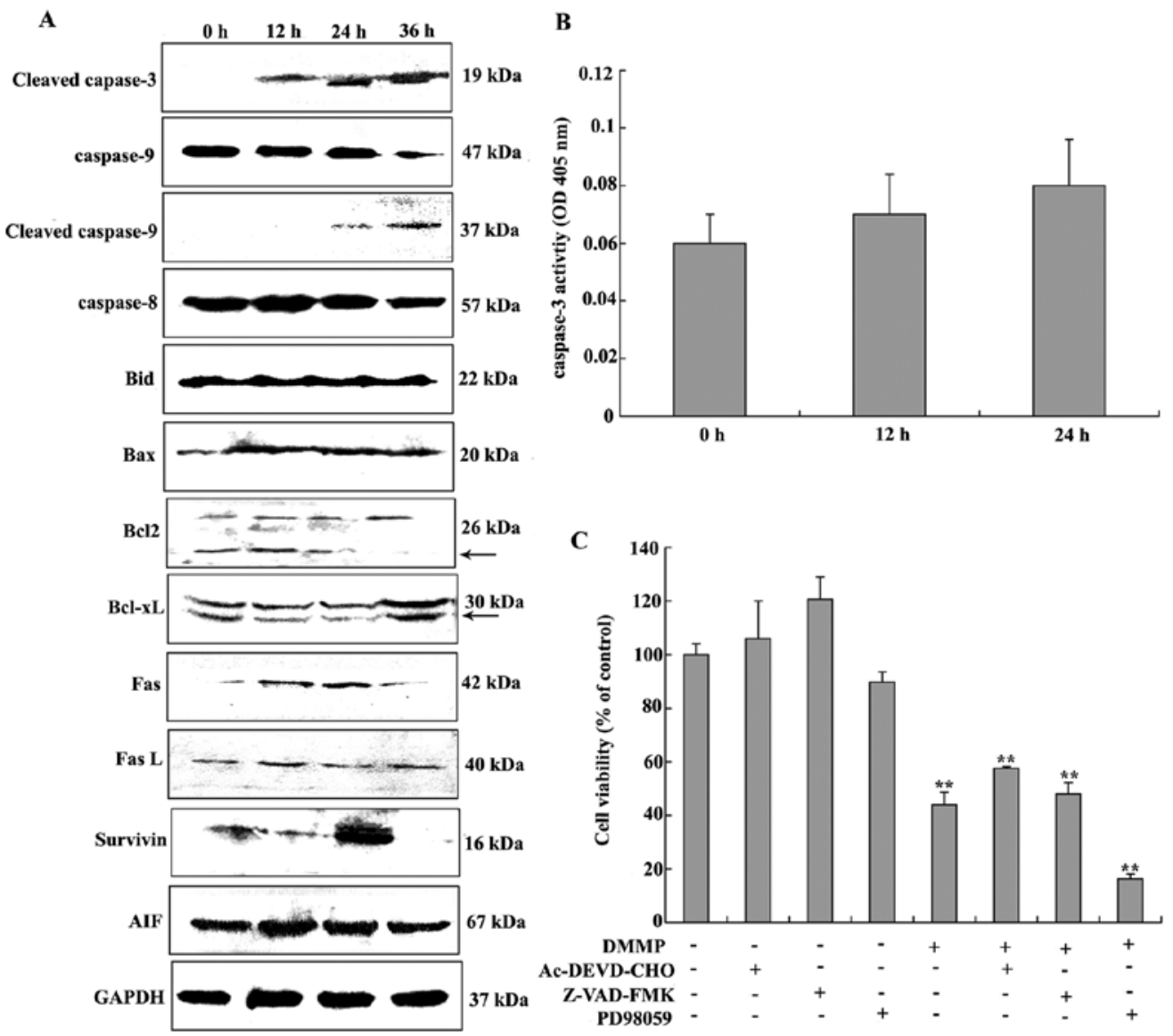

Figure 5. Activation of caspases induced by DMMP. (A) HeLa cells were treated with or without $40 \mu \mathrm{g} / \mathrm{ml}$ DMMP for different time periods. The cells were harvested and proteins were separated by SDS-PAGE and transferred onto PVDF membranes. The expression of apoptosis-related proteins was detected by western blotting probed with cleaved caspase-3, -9 and -8, Bid, Bax, Bcl-2, Bcl-xL, Fas, FasL, survivin, AIF and GAPDH antibodies. GAPDH was used as a loading control for western blotting. Arrowheads indicate the non-specific bands. (B) Activation of caspase-3 by DMMP. HeLa cells were incubated with $40 \mu \mathrm{g} / \mathrm{ml}$ DMMP for 12 and $24 \mathrm{~h}$ before the caspase-3 substrate Ac-DEVD-pNA was added. Incubation of the plate was carried out at $37^{\circ} \mathrm{C}$ for $2 \mathrm{~h}$, and the optical density (OD) was read on a fluorescence microplate reader. (C) Effects of caspase inhibitors and an ERK inhibitor on apoptosis induced by DMMP. HeLa cells were pretreated with the broad-spectrum caspase inhibitor (Z-VAD-FMK) and caspase-3 inhibitor (Ac-DEVD-CHO) at $10 \mu \mathrm{M}$ and ERK inhibitor (PD98059) at $20 \mu \mathrm{M}$ for $2 \mathrm{~h}$ followed by incubation with $40 \mu \mathrm{g} / \mathrm{ml}$ DMMP for $24 \mathrm{~h}$. The cell viability was evaluated using the MTT assay. Error bars represent \pm SD from triplicate independent experiments at each time point compared to the control group; ${ }^{*} \mathrm{P}<0.05,{ }^{* *} \mathrm{P}<0.01$.

at $12 \mathrm{~h}$ (2.5-fold ) and $24 \mathrm{~h}$ (2.2-fold), and then decreased at $36 \mathrm{~h}$ after treatment consistent with the results of the western blotting. Meanwhile, a pro-apoptotic Bcl-2 family member, Bax, was significantly increased (Fig. 5A). The mRNA expression levels of PUMA, NOXA, Bax, Bad and Bid were significantly upregulated after treatment (Fig. 7). The mRNA expression levels of PUMA and NOXA were significantly increased (3.4- and 5.4-fold, respectively) compared with the control at $12 \mathrm{~h}$ and achieved a maximum increase at $36 \mathrm{~h}$ after treatment (10.1- and 11.2-fold). Bax and Bid mRNA expression was significantly increased at $24 \mathrm{~h}$ after treatment (2.0- and 1.8-fold, respectively). Bim and Bad mRNA expression was significantly increased only at $36 \mathrm{~h}$ (3.5- and 3.6-fold, respectively).

Effect of DMMP on $p 53$ and $p 73$ expression. To investigate whether DMMP has an effect on p53 protein expression, we determined the p53 levels in HeLa cells treated with DMMP at the indicated time periods. Western blot analysis showed that DMMP treatment resulted in a time-dependent accumulation of p53 in HeLa cells (Fig. 8). Another p53 family member, p73, was also increased. Moreover, HeLa cells contain HPV E6-E7 genes which promote the degradation of $\mathrm{p} 53$ and the retinoblastoma protein. HPV E6-E7 mRNA levels were decreased by $50 \%$ following the drug treatment (Fig. 7 ).

Effect of DMMP on ERK and Akt. Western blot analysis showed that treatment with DMMP led to a slight upregulation of p-ERK in the HeLa cells following treatment while levels of total extracellular signal-regulated protein kinases 1 and 2 (ERK1/2) were not altered (Fig. 9). To further demonstrate the functions of ERK in inducing apoptosis, the effects of the highly specific ERK inhibitor PD98059 were examined. As shown in Fig. 5C, PD98059 significantly increased cell death. This indicates that sustained phosphorylation of ERK inhibits DMMP-induced cell death.

Akt is a serine/threonine kinase involved in cellular survival pathways by inhibiting apoptotic processes. To determine whether Akt is involved in DMMP-induced apoptosis, the phosphorylation status of Akt was examined by western blot analysis. Akt phosphorylation was significantly increased at $12 \mathrm{~h}$ and declined at $36 \mathrm{~h}$, while the level of total Akt protein was not altered (Fig. 9). 


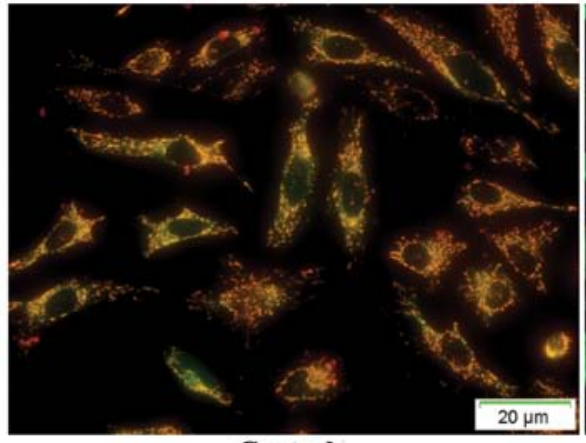

Control

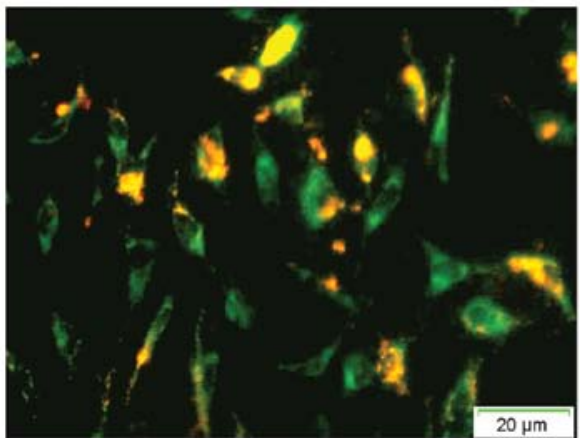

DMMP 12 h

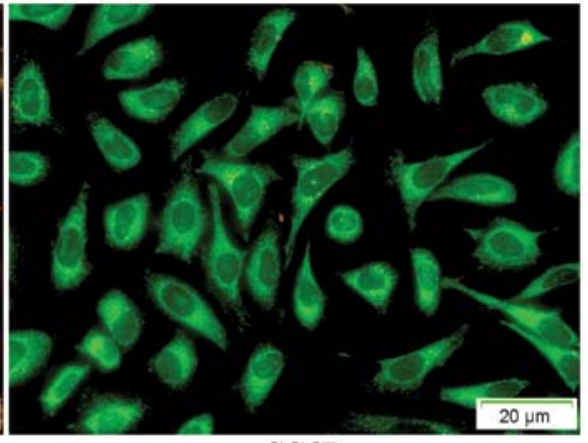

CCCP

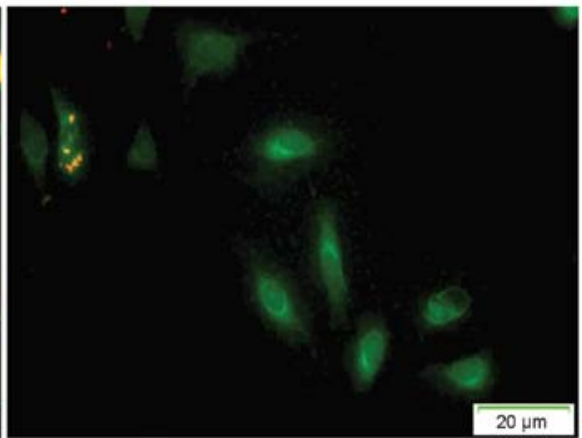

DMMP 24 h

Figure 6. DMMP induces depletion of mitochondrial membrane potential $(\Delta \Psi \mathrm{m})$. HeLa cells were treated with or without $40 \mu \mathrm{g} / \mathrm{ml}$ DMMP for the indicated time periods. HeLa cells were treated with $50 \mu \mathrm{M}$ CCCP for $20 \mathrm{~min}$ as the positive control. Disruption of $\Delta \Psi \mathrm{m}$ was observed by JC-1 staining. JC-1 spontaneously showed intense red fluorescence in the control cells with high $\triangle \Psi \mathrm{m}$. Cells were treated with CCCP; JC-1 showed only green fluorescence. Red fluorescence was diminished and green fluorescence was increased in HeLa cells treated with $40 \mu \mathrm{g} / \mathrm{ml}$ DMMP.

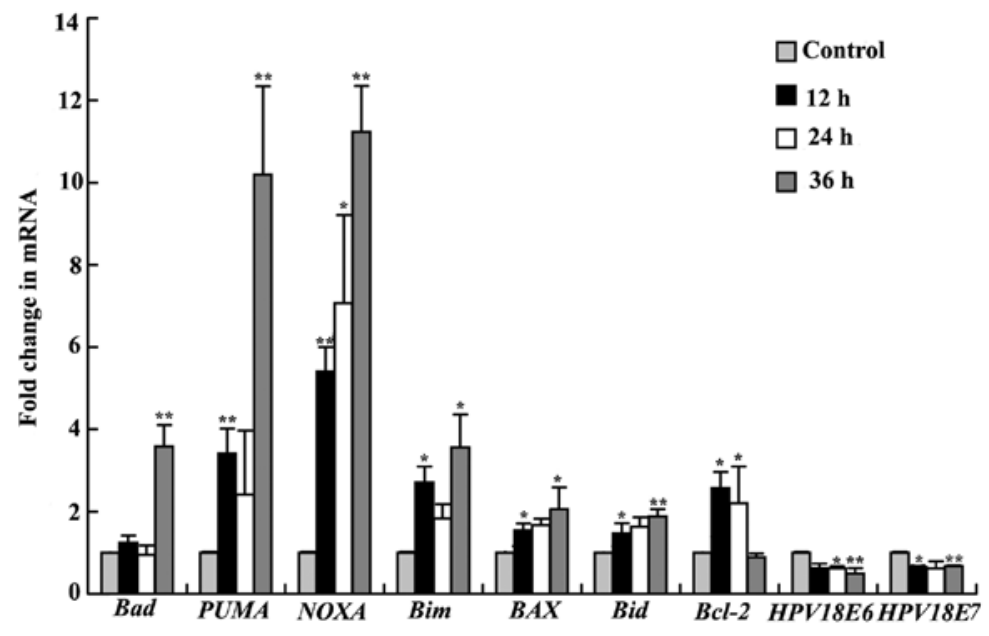

Figure 7. Real-time RT-PCR analysis of the expression of Bcl-2 family genes and HPV E6-E7 genes. HeLa cells were treated with $40 \mu \mathrm{g} / \mathrm{ml} \mathrm{DMMP}$ for 0,12 , 24 and $36 \mathrm{~h}$. RNA was prepared as described in Materials and methods. GAPDH was used as a control for RT-PCR. Error bars represent \pm SD from triplicate independent experiments at each time point compared to the control group; ${ }^{*} \mathrm{P}<0.05,{ }^{* *} \mathrm{P}<0.01$.

\section{Discussion}

During a search for novel anticancer agents in the culture broth of various endophytic fungi, DMMP was isolated from the endophytic fungus Pestalotiopsis photiniae. In the present study, DMMP inhibited the proliferation of various cancer cell lines and had little effect on normal lung MRC5 cells. As compared with the tumor cell lines analyzed, we found that DMMP had selective cytotoxic effect. Thus, the molecular mechanism of the cytotoxic effect of DMMP was investigated in HeLa cells.

DMMP caused cell cycle arrest in the G1 phase of the cell cycle. DMMP significantly elevated p27 ${ }^{\mathrm{KIP} 1}$ expression (Fig. 3). p2 $7^{\mathrm{KIP} 1}$ causes cell cycle arrest in G1 phase by binding cyclin E-CDK2 complexes (20-22). After DMMP treatment, phospho-Rb was slightly elevated, and E2F1 expression was significantly increased at 12 and $24 \mathrm{~h}$. Meanwhile, CDK2 and cyclin E proteins were also increased (Fig. 3B). 


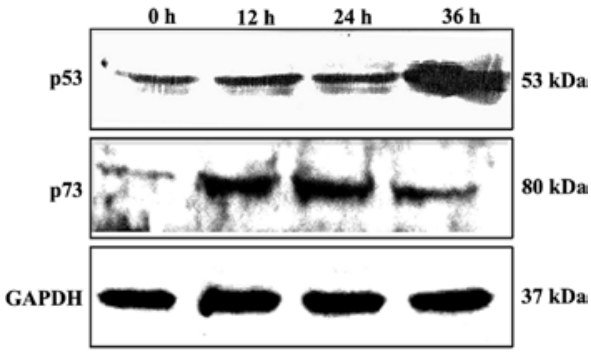

Figure 8. Effect of DMMP on the expression of the p53 family. Western blot analysis of expression levels of p53 and p73 following the exposure of $\mathrm{HeLa}$ cells to DMMP for $0,12,24$ and $36 \mathrm{~h}$. The cells were harvested and proteins were separated by SDS-PAGE and transferred onto a PVDF membrane. Western blot analysis was performed using specific antibodies for $\mathrm{p} 53, \mathrm{p} 73$ and GAPDH. GAPDH was used as a loading control.

The retinoblastoma protein $(\mathrm{Rb})$ exhibits growth inhibitory activity by binding with E2F1. The Rb-E2F1 pathway plays an important role in the regulation of cell cycle progression. Cyclin E-CDK2 can phosphorylate Rb resulting in the activation of E2F-responsive genes (23). Whether the increased levels of CDK2 and cyclin E proteins facilitated E2F function requires further investigation.

DMMP efficiently induced HeLa cell apoptosis characterized by compaction and fragmentation of nuclear chromatin. To further confirm the effects on apoptosis, FITC Annexin V and PI double staining and flow cytometry were performed. Results showed that DMMP induced apoptosis in the HeLa cells. Depletion of $\Delta \Psi \mathrm{m}$ in HeLa cells was also detected after treatment. The mitochondrial apoptotic pathway is mainly regulated by Bcl- 2 family proteins. Any imbalance in the expression level of pro-apoptotic and anti-apoptotic Bcl-2 members leads to the disruption of the outer mitochondrial membrane $(24,25)$. DMMP significantly upregulated the mRNA levels of pro-apoptotic genes such as Bax, PUMA, NOXA, Bim, Bid and Bad (3- to 11-fold) (Fig. 7). Meanwhile, $\mathrm{Bcl}-2$ and $\mathrm{Bcl}-\mathrm{xL}$ levels were decreased. Bax protein was significantly increased (Fig. 5A), and Bcl-2 mRNA expression was increased by 2 -fold and declined at $36 \mathrm{~h}$. As a result of these changes, the ratios of anti-apoptotic proteins and pro-apoptotic proteins of the Bcl-2 family were significantly reduced during apoptosis. The imbalance led to the loss of $\Delta \Psi \mathrm{m}$ after DMMP treatment.

The extrinsic apoptosis pathway is indicated by the ligation of cell surface death receptors to their specific ligands such as Fas/FasL (26). The formation of active caspase-8 is involved in the extrinsic apoptosis pathway. Extrinsic apoptosis in some cells is dependent on the cleavage of Bid. In our study, although FasL was slightly increased, we did not detect the activation of caspase- 8 and the truncated form of Bid (Fig. 5A). These results showed that DMMP-induced apoptosis did not occur through the Fas/FasL extrinsic apoptosis pathway.

Sequential activation of caspases plays a central role in the execution-phase of cell apoptosis. As an effector caspase, caspase-3 plays a central role in the extrinsic apoptotic pathway and intrinsic apoptotic pathway. In our study, although cleaved caspase- 3 and cleaved caspase- 9 were detected by western blotting, no significant activation of caspase-3 proteolytic activity was detected after drug treatment. Survivin, which

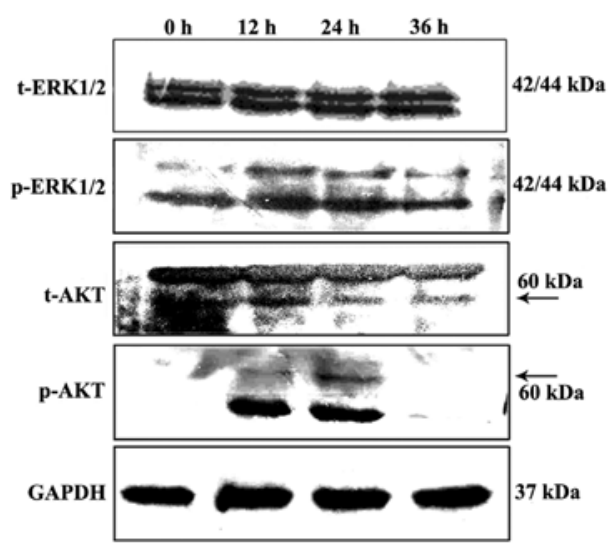

Figure 9. Effect of DMMP on the phosphorylation of ERK and AKT. HeLa cells were treated with $40 \mu \mathrm{g} / \mathrm{ml}$ DMMP for the indicated time periods. ERK1/2 and AKT and their phosphorylated forms were evaluated by western blotting using specific antibodies for p-AKT (Thr308), AKT, p-ERK1/2 (Thr202/Tyr204) and ERK1/2. Arrows indicate the non-specific bands. GAPDH was used as a loading control.

functions to inhibit caspase activation, was elevated after drug treatment. Elevated survivin may inhibit caspase-3 proteolytic activity. Further experiments showed that pretreatment with caspase inhibitors (Ac-DEVD-CHO and Z-VAD-FMK) failed to attenuate DMMP-induced cell death (Fig. 5C). DMMP induced a slightly higher level of AIF after drug treatment compared with the control (Fig. 5A). Although caspase activation is considered a hallmark of apoptotic cell death, caspase-independent apoptosis has been reported. Apoptosisinduced factors (AIF) and endonuclease $\mathrm{G}$ (EndoG) residing in mitochondria are involved in the caspase-independent cell death pathway (27). DMMP may induce apoptosis in a caspase-independent manner. Further experimental investigation is needed for confirmation.

The tumor suppressor transcription factor $\mathrm{p} 53$ plays a vital role in cell cycle arrest and apoptosis in response to cellular stress. Western blot analysis showed that DMMP treatment resulted in a significant accumulation of $\mathrm{p} 53$ and $\mathrm{p} 73$ proteins in HeLa cells (Fig. 8). Normally, p53 protein is rapidly degraded by the ubiquitin-proteome system. $\mathrm{Mdm} 2$ is an important negative regulator of the p53 tumor suppressor $(28,29)$. But in HPV-infected cervical cancer cells, HPV E6 is the protein responsible for repressing p53 replacing $\mathrm{Mdm} 2$ function (30). In the present study, real-time RT-PCR showed that DMMP caused a $\sim 50 \%$ reduction of E6 and E7 mRNA expression when compared to the control (Fig. 7). The dramatic upregulation of p53 protein may be due to the low expression of HPV E6.

In large part, the potent anticancer activity of p53 has been linked to its ability to induce apoptosis through the intrinsic mitochondrial-mediated apoptotic pathway (31). p53 enhances the expression of Bcl-2 family members including Bax, Bid, PUMA and Noxa (32). BH3-only proteins, NOXA and PUMA, are important mediators of p53-induced apoptosis (33). E2F1 is the first member of the E2F transcription factor family. Evidence shows that E2F1 also possesses tumor suppressor functions which can induce apoptosis via p53-dependent and p53-independent pathways (34). Oncogenic signaling by E2F1 has recently been linked to stabilization and activation of the tumor suppressor p53 (35). Puma and Noxa can 
be regulated by both p53 and E2F1. In our study, E2F1 and p53 were both increased after DMMP treatment. The mRNA levels of Bax, Puma, Noxa and Bid genes were significantly increased (Fig. 7). Whether p53 and E2F1 were involved in DMMP-induced apoptosis requires further investigation using p53 or E2F1 knockdown cells.

Extracellular signal-regulated protein kinases 1 and 2 (ERK1/2) are members of the mitogen-activated protein kinase superfamily that can mediate cell proliferation and apoptosis (36). PhosphoERK1/2 was slightly increased after DMMP treatment (Fig. 8). Treatment with the ERK inhibitor PD98059 and DMMP efficiently induced HeLa cell death. This implied that DMMP combined with PD98059 may represent a novel anticancer strategy. The Akt serine/threonine kinases are critical regulators of cell survival. The activation of the PI3K-Akt pathways promotes tumorigenesis by inhibiting apoptotic processes (37). Following DMMP treatment, phospho-Akt, increased at 12 and $24 \mathrm{~h}$, was slightly detected at $36 \mathrm{~h}$ while total Akt was unaltered (Fig. 9). The reason for the elevated phosphorylation of Akt at an early stage requires further investigation.

In conclusion, for the first time we demonstrated that DMMP significantly induced HeLa cell apoptosis and caused cell cycle arrest in G1 phase. We investigated the possible mechanisms involved in DMMP-induced apoptosis. We demonstrated that DMMP-induced apoptosis was through the mitochondrial intrinsic pathway in HeLa cells. DMMP upregulated p27 protein, and caused cell cycle arrest in the G1 phase. The p53 family members, p53 and p73, were increased after drug treatment. Our results indicate that DMMP is a promising cancer therapeutic agent.

\section{Acknowledgements}

This study was supported by programs for New Century Excellent Talents in University (NCET-09-0112), the National Natural Science Foundation of China (30901755 and 31171885), the Scientific Research Program of Hebei Provincial Education Bureau (2011107), the Hebei Province Science Fund for Distinguished Young Scholars (C2011201113), and the Program for Changjiang Scholars and Innovative Research Team in the University (IRT1124).

\section{References}

1. Koehn FE and Carter GT: The evolving role of natural products in drug discovery. Nat Rev Drug Discov 4: 206-220, 2005.

2. Miller KI, Qing C, Sze DM and Neilan BA: Investigation of the biosynthetic potential of endophytes in traditional Chinese anticancer herbs. PLoS One 7: e35953, 2012.

3. Tan RX and Zou WX: Endophytes: a rich source of functional metabolites. Nat Prod Rep 18: 448-459, 2001.

4. Reed JC: Drug insight: cancer therapy strategies based on restoration of endogenous cell death mechanisms. Nat Clin Pract Oncol 3: 388-398, 2006.

5. Frankfurt OS and Krishan A: Apoptosis-based drug screening and detection of selective toxicity to cancer cells. Anticancer Drugs 14: 555-561, 2003.

6. Chen F, Wang W and El-Deiry WS: Current strategies to target p53 in cancer. Biochem Pharmacol 80: 724-730, 2010.

7. Collavin L, Lunardi A and Del Sal G: p53-family proteins and their regulators: hubs and spokes in tumor suppression. Cell Death Differ 17: 901-911, 2010.

8. Ferreira CG, Tolis C and Giaccone G: p53 and chemosensitivity. Ann Oncol 10: 1011-1021, 1999.
9. Lunghi P, Costanzo A, Mazzera L, Rizzoli V, Levrero M and Bonati A: The p53 family protein p73 provides new insights into cancer chemosensitivity and targeting. Clin Cancer Res 15: 6495-6502, 2009.

10. Bisso A, Collavin L and Del Sal G: p73 as a pharmaceutical target for cancer therapy. Curr Pharm Des 17: 578-590, 2011.

11. Slade N and Horvat A: Targeting p73 - a potential approach in cancer treatment. Curr Pharm Des 17: 591-602, 2011.

12. Lain S, Hollick JJ, Campbell J, et al: Discovery, in vivo activity, and mechanism of action of a small-molecule p53 activator. Cancer Cell 13: 454-463, 2008.

13. Vassilev LT, Vu BT, Graves B, et al: In vivo activation of the p53 pathway by small-molecule antagonists of MDM2. Science 303: 844-848, 2004

14. Peirce SK and Findley HW: The MDM2 antagonist nutlin-3 sensitizes p53-null neuroblastoma cells to doxorubicin via E2F1 and TAp73. Int J Oncol 34: 1395-1402, 2009.

15. Sampath D, Calin GA, Puduvalli VK, et al: Specific activation of microRNA106b enables the p73 apoptotic response in chronic lymphocytic leukemia by targeting the ubiquitin ligase Itch for degradation. Blood 113: 3744-3753, 2009.

16. Phuwapraisirisan P, Rangsan J, Siripong P and Tip-Pyang S: New antitumour fungal metabolites from Alternaria porri. Nat Prod Res 23: 1063-1071, 2009.

17. Suemitsu R, Ohnishi K, Morikawa Y and Nagatomo S: Zinnimidine and 5-(3',3'-dimethylallyloxy)-7-methoxy-6-methylphthalide from Alternaria porri. Phytochemistry 38: 495-497, 1995.

18. Yang XL, Zhang S, Hu QB, Luo DQ and Zhang Y: Phthalide derivatives with antifungal activities against the plant pathogens isolated from the liquid culture of Pestalotiopsis photiniae. J Antibiot (Tokyo) 64: 723-727, 2011.

19. Malumbres M and Barbacid M: To cycle or not to cycle: a critical decision in cancer. Nat Rev Cancer 1: 222-231, 2001.

20. Hengst L and Reed SI: Translational control of $\mathrm{p} 27^{\mathrm{KIP} 1}$ accumulation during the cell cycle. Science 271: 1861-1864, 1996.

21. Pagano M, Tam SW, Theodoras AM, et al: Role of the ubiquitin-proteasome pathway in regulating abundance of the cyclin-dependent kinase inhibitor p27. Science 269: 682-685, 1995.

22. Vervoorts $\mathbf{J}$ and Lüscher B: Post-translational regulation of the tumor suppressor p27(KIP1). Cell Mol Life Sci 65: 3255-3264, 2008.

23. Harbour JW and Dean DC: The Rb/E2F pathway: expanding roles and emerging paradigms. Genes Dev 14: 2393-2409, 2000.

24. Danial NN and Korsmeyer SJ: Cell death: critical control points Cell 116: 205-219, 2004

25. Chipuk JE and Green DR: How do BCL-2 proteins induce mitochondrial outer membrane permeabilization? Trends Cell Biol 18: 157-164, 2008.

26. Ashkenazi A and Dixit VM: Death receptors: signaling and modulation. Science 281: 1305-1308, 1998.

27. Chipuk JE and Green DR: Do inducers of apoptosis trigger caspase-independent cell death? Nat Rev Mol Cell Biol 6: 268-275, 2005

28. Brooks CL and $\mathrm{Gu} \mathrm{W}: \mathrm{p} 53$ ubiquitination: $\mathrm{Mdm} 2$ and beyond. Mol Cell 21: 307-315, 2006.

29. Sullivan KD, Gallant-Behm CL, Henry RE, Fraikin JL and Espinosa JM: The p53 circuit board. Biochim Biophys Acta 1825: 229-244, 2012.

30. Diaz D, Santander MA and Chavez JA: HPV-16 E6 and E7 oncogene expression is downregulated as a result of $\mathrm{Mdm} 2$ knockdown. Int J Oncol 41: 141-146, 2012

31. Chipuk JE and Green DR: Dissecting p53-dependent apoptosis. Cell Death Differ 13: 994-1002, 2006.

32. Hemann MT and Lowe SW: The p53-Bcl-2 connection. Cell Death Differ 13: 1256-1259, 2006.

33. Villunger A, Michalak EM, Coultas L, et al: p53- and drug-induced apoptotic responses mediated by $\mathrm{BH} 3$-only proteins puma and noxa. Science 302: 1036-1038, 2003.

34. Wu Z, Zheng S and Yu Q: The E2F family and the role of E2F1 in apoptosis. Int J Biochem Cell Biol 41: 2389-2397, 2009.

35. Stiewe T and Pützer BM: Role of the p53-homologue p73 in E2F1-induced apoptosis. Nat Genet 26: 464-469, 2000.

36. Mebratu Y and Tesfaigzi Y: How ERK1/2 activation controls cell proliferation and cell death: is subcellular localization the answer? Cell Cycle 8: 1168-1175, 2009.

37. Amin AR, Paul RK, Thakur VS and Agarwal ML: A novel role for p73 in the regulation of Akt-Foxola-Bim signaling and apoptosis induced by the plant lectin, Concanavalin A. Cancer Res 67: 5617-5621, 2007. 